\title{
Treinamento resistido para crianças e adolescentes
}

\section{Resistance training for children and adolescents}

Jucemar Benedet ${ }^{1,2}$, Juliana Carla Freddi $^{2}$, Alexandre Paiva Luciano ${ }^{3}$, Fernando de Souza Almeida ${ }^{3}$, Gilvan Lira da Silva ${ }^{3}$, Patrícia de Fragas Hinnig ${ }^{4}$, Fernando Adami ${ }^{3}$

${ }^{1}$ Programa de Pós-graduação em Educação Física - UFSC - Florianópolis (SC), Brasil.

${ }^{2}$ Curso de Graduação em Educação Física - Universidade do Sul de Santa Catarina (UNISUL) - (SC), Brasil.

${ }^{3}$ Departamento de Saúde da Coletividade - Faculdade de Medicina do ABC (FMABC) - Santo André (SP), Brasil.

${ }^{4}$ Departamento de Epidemiologia - Faculdade de Saúde Pública da Universidade de São Paulo (FSP/USP) - São Paulo (SP), Brasil.

DOI: http://dx.doi.org/10.7322/abcshs.v38i1.7

\section{RESUMO}

Em uma sociedade onde a aparência corporal é muito valorizada, as academias de musculação são procuradas de forma cada vez mais precoce pelos jovens. O objetivo deste estudo foi fazer uma análise retrospectiva sobre a evolução das recomendações do treinamento resistido para crianças e adolescentes. A busca bibliográfica foi realizada nas bases de dados PubMed, Web of Science e Scielo. As estratégias de busca incluíram a combinação dos seguintes descritores: "resistance training", "strength training", "weight-training" "adolescents", "children"e "youth". A busca bibliográfica resultou em 349 artigos. A leitura e análise dos resumos pelos autores resultou na seleção de 25 artigos, respeitando os critérios de inclusão adotados. Os resultados indicam que as primeiras publicações sobre o tema ocorreram em 1990 e, a partir de então, observou-se uma evolução substancial tanto no número de publicações quanto nas abordagens do treinamento resistido para crianças e adolescentes. A análise dos artigos mostra que atualmente é possível encontrar informações qualificadas cujos indicativos suportam a prática do treinamento resistido por parte de crianças e adolescentes de forma segura e eficaz. Pode-se concluir que não restam dúvidas sobre os benefícios do treinamento resistido para a saúde dos jovens, respeitadas as recomendações vigentes as quais são pontuadas ao longo desta revisão. Convém destacar que permanecem latentes questões associadas às respostas crônicas do treinamento resistido nesta faixa etária, assim como dúvidas em relação à prescrição para as diferentes idades, ergonomia dos equipamentos e ambientes.

Palavras-chave: treinamento de resistência; treinamento; atividade motora; adolescente.

\begin{abstract}
In a society where personal appearance is highly valued, the gyms are increasingly sought early by young people. The aim of this study was to conduct a retrospective analysis about the evolution of resistance training recommendations for children and adolescents. A literature search was held in the databases PubMed, Web of Science and Scielo. The search strategies included the combination of the following keywords: "resistance training", "strength training", "weight-training" "adolescents", "children" and "youth". The literature search resulted in 349 articles. The reading and analysis of the abstracts by authors resulted in the selection of 25 articles, respecting the criteria adopted. The results indicate that the first publications took place in 1990 and, from then on, there was a substantial evolution in the number of publications and in the approaches of resistance training for children and adolescents. The analysis of the articles shows that it is currently possible to find qualified information whose indications support a safely and an effectively practice of resistance training for children and adolescents. It can be concluded that there is no doubt about the benefits of resistance training for the health of young people, respected the recommendations in force which are peppered throughout this review. It might want to highlight that remain latent issues associated to chronic responses of resistance training in this age group, as well as doubts regarding the prescription for different ages, ergonomics of equipment and environments.
\end{abstract}

Keywords: resistance training; training; motor activity; adolescent. 


\section{INTRODUÇÃO}

Em uma sociedade onde a aparência corporal é muito valorizada, a procura dos jovens pelas academias de musculação é cada vez mais precoce. Ainda que estejam bem documentados os benefícios das atividades físicas na infância, a busca por uma imagem corporal, muitas vezes idealizada pelos pais, mídia, grupos sociais e pelos próprios adolescentes, pode desencadear comportamentos maléficos à saúde ${ }^{1}$. Normalmente, atividades cujas condições de volume e intensidade são elevados aliados a déficit nutricional ou uso de substâncias ilícitas, podem comprometer a saúde. Este comprometimento pode surgir por meio de lesões, alterações no ciclo maturacional ou de crescimento. Neste contexto, está bem estabelecido que um dos principais fatores prejudiciais associado ao treinamento intenso, não exclusivo ao treinamento resistido (TR), é a inibição do eixo GH-IGF1 que pode comprometer o crescimento longitudinal de crianças e adolescentes ${ }^{2}$.

O exercício físico, administrado de forma equivocada, pode acarretar lesões musculares, ósseas e articulares. Porém, há relatos de que lesões em crianças e adolescentes derivadas do TR são pouco comuns e numericamente semelhantes aos adultos. As mais frequentes parecem ser os estiramentos e as distensões musculares, assim como lesões por excesso de treinamento ${ }^{3} \mathrm{Na}$ literatura, não é relatado déficit estatural devido ao treinamento resistido, respeitadas às condições de volume, intensidade e aporte energético ${ }^{4,5}$.

Em contrapartida, estudos têm mostrado que a prática de exercícios resistidos por crianças e adolescentes pode melhorar indicadores de saúde tais como a composição corporal, o sistema cardiovascular, o perfil lipídico, a densidade mineral óssea e aumentar a produção sérica do fator de crescimento IGF- ${ }^{6-8}$. Recentes estudos tem destacado as respostas positivas do exercício físico sobre importantes mediadores do tecido adiposo tais como a leptina. Este hormônio tem uma estreita relação com o metabolismo e por consequência com as doenças degenerativas, oriundas principalmente do excesso de gordura corporal ${ }^{9,10}$. Neste mesmo sentido, a prática de atividades físicas interfere de forma benéfica sobre a homeostase dos compartimentos corporais, os quais tem se mostrado associado a densidade mineral óssea $^{11}$. Portanto, o TR é um dos componentes relevantes para um bom condicionamento físico, porém outros fatores, tais como a alimentação e aspectos relacionados ao estilo de vida, têm igual importância ${ }^{12}$.

Os termos treinamento resistido, treinamento de força e levantamento de peso são usados normalmente como sinônimos. Entende-se que o termo "treinamento resistido" abrange um alcance mais amplo de modalidades de treinamento e uma maior amplitude de expectativas de treinamento ${ }^{13}$. O treinamento de força é definido como o uso de métodos de resistência para aumentar a capacidade para exercer ou resistir à força ${ }^{14}$. O treinamento pode utilizar pesos livres, o próprio peso do corpo do indivíduo, máquinas, bandas elásticas ou outros dispositivos de resistência para atingir esta meta ${ }^{4,15}$.
Mais recentemente, com a crescente inovação em relação aos métodos de treinamento de força, surgiu o conceito de "Core strengthening", ou seja, fortalecimento do centro do corpo. Este treinamento enfatiza fortalecer a musculatura abdominal e dos glúteos, assim como desenvolver a flexibilidade de anexos musculares à pelve, tal como o quadríceps ${ }^{15}$. Em função de suas qualidades, esta vertente do treinamento resistido juntamente com os exercícios funcionais tem sido uma das estratégias metodológicas mais propagadas para crianças e adolescentes. Nos Estados Unidos da América, o número de academias e clubes para crianças de seis a dezessete anos continua crescendo exponencialmente, pois há um consenso de que o treinamento de resistência seguro pode melhorar a saúde e a aptidão física dos joven ${ }^{13}$.

Apesar do aumento do número de publicações sobre este tópico, o treinamento resistido para crianças e adolescentes permanece controverso e com lacunas ${ }^{16}$. Assim, o objetivo deste estudo de revisão foi fazer uma análise retrospectiva a partir de consensos e revisões da literatura sobre a prescrição de exercícios resistidos para crianças e adolescentes. Pretende-se destacar o processo de evolução relativo às recomendações, estabelecer um consenso atual com base nos estudos revisados, bem como encontrar possíveis lacunas em relação ao tema proposto.

\section{MÉTODO}

A busca bibliográfica foi realizada nas bases de dados PubMed (U.S National Library of Medicine), Web of science, e Scielo (Scientific Electronic Library Online). No PubMed e Web of science foram utilizados como descritores de busca os termos "resistance training in children and adolescents"; "strength training in children and adolescents"; "resistance training in children and youth" $e$ "weight-training in children and adolescents". No Scielo foram utilizadas as palavras-chave: "musculação e crianças", "musculação e adolescentes" "treinamento resistido e crianças" e "treinamento resistido e adolescentes". Utilizou-se uma variedade maior de descritores devido à heterogeneidade relativa à nomenclatura deste tipo de treinamento e a pouca quantidade de estudos relacionados à temática proposta. A pesquisa foi realizada no período de 29 a 31 de março de 2011. Com o intuito de maximizar a especificidade da busca e facilitar a triagem das informações, na base Pubmed foram impostos os limites "título e resumo" e "disponibilidade do resumo". Na base Web of science, a busca foi limitada para artigos em todas as bases de dados (SCI-EXPANDED, SSCI, A\&HCI), sem limites para data de publicação. No Scielo utilizou-se o formulário livre e no campo de busca "todos os índices". Em todas as bases de dados não se optou por incluir somente revisões com a intenção de capturar consensos de especialistas que por ventura não estivessem classificados como revisões.

Para serem inclusos no estudo os artigos deveriam ser necessariamente de revisão bibliográfica ou consensos de especialistas que analisaram diretamente a prescrição e o impacto dos exercícios resistidos em crianças e adolescentes com relação à aptidão 
física relacionada a saúde. Justifica-se a adoção desta estratégia em três motivos principais: a) as principais informações sobre o tema ao longo das últimas décadas estão sintetizadas nas revisões e consensos; b) são poucos os estudos de intervenção e/ou experimentais voltados à aptidão física relacionada à saúde na faixa etária em questão, além da dificuldade na comparação, aja vista as diferenças metodológicas; c) entende-se que a qualidade e a robustez das informações em revisões e consensos sirvam de parâmetro para os demais estudos. Artigos de revisão que foram relacionados ao treinamento resistido em atletas ou em patologias específicas foram desconsiderados, bem como os artigos sem seus resumos online. Para seleção e inclusão dos estudos, os resumos e artigos foram lidos e avaliados por dois pesquisadores de forma independente.

\section{RESULTADOS}

A busca bibliográfica resultou em 349 artigos, sendo 122 da base Medline/Pubmed, 224 da base Web of science e três da base de dados Scielo. Retiradas as referências cruzadas redundantes $(\mathrm{n}=74)$ foram selecionados 275 artigos. Um total de 250 trabalhos não foram selecionados, sendo que destes, 179 não eram artigos de revisão ou consensos de especialistas, 55 tratavam de treinamento resistido para jovens atletas e/ou com patologias específicas e 16 não disponibilizaram o resumo. Ao final, foram incluídos 24 artigos das bases PubMed e Web of Science e um artigo da base Scielo, totalizando 25 artigos elegíveis para o estudo. Quinze artigos estavam disponíveis diretamente no portal da CAPES e dez foram solicitados aos autores, via correio eletrônico. Em relação à característica dos estudos 19 são estudos de revisão, dois são metanálises e quatro são consensos ou posicionamentos de associações publicados pela American Academy of Pediatrics por meio do seu Committee on Sports Medicine and Fitness, pela Canadian Society for Exercise Physiology e pela National Strength and Conditioning Association (NCSA). Os principais achados indicam que no início dos anos de 1990 a maior preocupação dos autores estava em esclarecer as questões de segurança e aplicabilidade do TR para crianças e adolescentes. Posteriormente passam a ser abordadas questões voltadas para a qualificação dos profissionais, segurança e saúde da criança e do adolescente associadas ao TR. Os artigos mais recentes abordam questões associadas à manutenção, diversificação e aderência do TR para jovens.

\section{DISCUSSÃO}

Os primeiros estudos datam de 1990 como sendo o período de início de publicações sobre o treinamento resistido (TR) para crianças e adolescentes. Nesta época, Webb ${ }^{17}$, em estudo de revisão, relata que o treinamento de força para crianças e adolescentes ainda encontra resistências na sociedade e não está totalmente livre de riscos. O autor descreve preocupações médicas relacionadas a lesões, a hipertensão e doenças correlacionadas. Sobre as lesões concluiu que os índices são provavelmente baixos, comparáveis a muitas atividades juvenis que são consideradas seguras. Por fim, sugere que a incidência e severidade das lesões provavelmente podem ser reduzidas por adesão às orientações de adequação de volume (moderado), intensidade (moderada a baixa) e tipos de exercícios propostos (estruturais).

Nesta mesma época e com uma perspectiva similar, Risser ${ }^{18,19}$ publicou duas revisões tendo como principais objetivos formular diretrizes para a prevenção de lesões músculo-esqueléticas derivadas do TR. Suas principais conclusões indicaram que, quando os jovens ficam expostos ao treinamento rigoroso com pesos, podem ocorrer lesões nos discos intervertebrais, espondilólise e lesões no joelho (menisco), porém tais lesões não são exclusivas da faixa etária em questão. No entanto, o autor não estabeleceu de forma definitiva uma relação inversa entre idade e risco de lesão. Ressalta que programas adequados, considerando a maturidade física e emocional, oferecem segurança e baixa probabilidade de lesões, tal qual na população adulta.

Em relação aos efeitos do TR em idades tenras, Blimkie ${ }^{20}$ destacou que este parece ter pouco ou nenhum efeito sobre o tamanho do músculo (hipertrofia), no caso dos pré-adolescentes. Os resultados de ganho de força estariam mais associados às funções neurais - ativação, integração, sincronização e recrutamento de unidades motoras. O TR nesta faixa etária, além dos ganhos de força, pode contribuir na coordenação motora e resistência geral, o que se traduz em benefícios diretos no crescimento físico e aptidão física. Segundo o autor, efeitos de hipertrofia são mais claramente observados no início da adolescência com o advento dos processos maturacionais, porém outros indicadores do condicionamento físico podem sofrer alterações significativas nesta faixa etária.

Em 1993, Blimkie ${ }^{21}$ retomou o discurso dos efeitos do TR para pré-adolescentes afirmando que em curto prazo o treinamento não parece ter nenhum efeito no crescimento somático (altura e peso), composição corporal e nenhuma influência positiva comprovada no desempenho atlético, índice de lesões e na recuperação de lesões. Em contraste, reforça que o risco de lesões com treinamento adequado e prudentemente prescrito praticamente inexiste. Dentro desta perspectiva, concluiu que o treinamento de resistência em curto prazo não traz nenhum efeito prejudicial durante a pré-adolescência em indicadores antropométricos, cardiorrespiratórios e na pressão arterial.

Em metanálise realizada por Payne ${ }^{22}$, constatou-se que o TR em jovens aumenta a força e a resistência muscular. Estes resultados podem variar por sexo, idade e tipo de treinamento. Tais diferenças se justificam, pois as alterações hormonais têm relação direta sobre a força e resistência muscular, em diferentes proporções para meninos e meninas. Sobre o tipo de treinamento, o autor ressaltou que o isotônico mostra-se mais efetivo do que o isométrico e o isocinético, nesta faixa etária.

Somente em 1998 foram descritas de forma detalhada as principais diferenças entre adultos e crianças em relação à prescrição 
do $\mathrm{TR}^{23}$. Crianças e jovens devem trabalhar em uma faixa de intensidade submáxima, número de repetições baixo, amplitude completa e exercitar-se com uma frequência de duas a três vezes na semana. Para o aumento de massa magra, o autor sugere uma a três séries, oito a doze repetições com quatro ou cinco grupos musculares, iniciando sem adição de peso. Se o objetivo for a redução do peso corporal, indica-se um número maior de repetições (nove a 15). Em ambos os casos, as sessões devem ser complementadas com treinamento cardiovascular e de flexibilidade. Para o autor, a supervisão é um componente vital e a chave do sucesso baseia-se em três componentes: técnica de execução, progressão adequada e equipamento seguro. As principais diferenças em relação ao treinamento de adultos dizem respeito à intensidade e volumes mais baixos sendo que questões relativas à metodologia ainda não se mostravam evidentes.

A partir do final da década de 1990, as publicações sobre o tema começaram a ganhar volumes mais significativos e abordagens mais diversificadas, indicando um nítido sinal do crescimento do TR entre os jovens. Faigenbaum ${ }^{24}$ em seu artigo de revisão, reforçou as questões de segurança e eficácia, destacando que os pais, professores, treinadores e profissionais da saúde devem compreender que o treinamento de força na juventude é um método especializado de condicionamento que pode oferecer benefícios. Corrobora com Alleyne ${ }^{23}$, em relação à instrução qualificada, supervisão competente e uma progressão apropriada a faixa etária, diferente do ritmo propagado entre adultos. Destaca a necessidade de estudos clínicos com crianças e adolescentes para melhor explorar os efeitos agudos e crônicos do TR.

Em 2001, a American Academy of Pediatrics, por meio do seu Committee on Sports Medicine and Fitness ${ }^{4}$ divulgou uma série de recomendações sobre TR para crianças e adolescentes para garantir sua segurança e eficácia, dentre elas: $i$ ) evitar o levantamento competitivo de peso, antes de iniciar um programa formal de TR; ii) avaliação por um pediatra ou médico do esporte; iii) realização de condicionamento aeróbico concomitante; $i v$ ) não utilização de carga adicional em exercícios de treinamento de força (somente o peso do corpo; $v$ ) realização de oito a 15 repetições para todos os grupos musculares com amplitude total dos movimentos; vi) avaliação ao primeiro sinal de lesão ou desconforto antes de continuar o exercício em questão.

Nesta época, o TR para jovens já era considerado popular em países como os Estados Unidos e Canadá, onde já se desenvolviam métodos para melhorar o desempenho atlético nesta faixa etária. Os estudos mostravam inclusive não haver perda de flexibilidade com o treinamento adequado e salientavam que os ganhos de força são em grande parte revogáveis quando o treinamento é suspendido, tal qual na população adulta ${ }^{2}$. Os resultados decorrentes do TR passaram a ter destaque também em relação à preparação atlética e suas especificidades em diversas modalidades esportivas. Em relação à aptidão física e saúde, a combinação com atividades aeróbicas passa a ser recomendada com maior ênfase ${ }^{25}$.

No ano de 2003, Falk e Eliakim ${ }^{7}$ relataram em seu estudo que haviam lacunas na literatura em relação à intensidade ideal e ao volume de treinamento para jovens de diferentes idades. Além disso, de acordo com os autores, os poucos estudos que tratavam destas variáveis, mostravam-se contrários ao falso juízo comum de que o TR pode retardar o crescimento. Encontraram evidências mais contundentes de que os fatores de crescimento (IGFs) são aumentados com o TR e que não há nenhum efeito prejudicial no crescimento linear quando prescrito de forma correta. Os achados deste estudo reforçaram a hipótese de que lesões e efeitos de inibição de fatores de crescimento estão associados ao treinamento exaustivo, mais presente entre jovens atletas do que na população em geral.

Benjamin e Glow ${ }^{26}$ focaram em seu artigo de revisão as questões que envolvem médicos pediatras e pais em relação às escolhas e expectativas de treinamento para jovens. Na perspectiva dos autores, os três componentes que incluem a supervisão adulta, o equipamento adequado e as expectativas realistas, tornam o treinamento para crianças e adolescentes seguro e eficaz, tendência já documentada em estudos anteriores.

Meyer e Wall ${ }^{27}$, além de recomendações para a escolha (exercícios estruturais) e intensidade (oito a doze repetições) dos exercícios, salientaram que a maturidade sexual é um importante fator a ser observado para prescrição adequada dos exercícios e prevenção de lesões. Apresentaram as vantagens e desvantagens do TR realizado com o peso do próprio corpo, com as máquinas e pesos livres. Demonstraram, por meio de fotos, tipos de exercícios alternativos a serem realizados pelos adolescentes, sempre com supervisão de um profissional.

Em revisão realizada por Malina ${ }^{5}$, foram analisados protocolos experimentais de treinamento com pesos e máquinas de resistência em jovens. Nos casos em que havia supervisão de adultos e cuja relação instrutor/participante foi baixa, os programas foram descritos como relativamente seguros e incapazes de influenciar negativamente no crescimento e maturação. Na revisão foram observados resultados de estudos que mostraram mudanças pouco significativas na composição corporal e nos ganhos significativos de força, caracterizando o TR como um importante coadjuvante no condicionamento físico dos jovens.

Esta mesma tendência foi verificada por Benson e colaboradores $^{28}$. Os autores investigaram os efeitos do treinamento de resistência na aptidão metabólica (gordura corporal, lipídios, insulina, glicose) de crianças e adolescentes menores de 18 anos. Nos 12 estudos incluídos, foi observada uma pequena adaptação positiva na saúde metabólica de crianças e adolescentes devido ao TR supervisionado, porém ainda não há descrito na literatura a extensão e duração de tais benefícios, devido às limitações metodológicas destes estudos. Portanto a participação de jovens no TR é uma alternativa a ser considerada não 
só na preparação para os esportes, mas também para a promoção e manutenção da aptidão física relacionada à saúde ${ }^{28}$. Neste contexto, Vaughn e Micheli ${ }^{29}$ destacaram a importância do acompanhamento constante do índice de progressão, pois nesta faixa etária não se pode esperar independência do aluno em relação a todas as variáveis do treinamento, principalmente aquela que envolve a determinação da carga de trabalho.

A American Academy of Pediatrics ${ }^{15}$, no ano de 2008, publicou novas recomendações sobre o TR para crianças e adolescentes que incluiram técnicas adequadas e precauções de segurança. Recomendam evitar o power lifting e body building; não utilizar substâncias que melhoram o desempenho e esteroides anabolizantes; crianças com doença cardíaca congênita devem consultar um cardiologista; e manutenção do condicionamento aeróbico juntamente com o treinamento de resistência. As novas recomendações incluíram ainda cuidados com a ingestão adequada de líquidos e alimentos, aquecimento metabólico, "supervisão adequada" com uma relação instrutor/aluno não mais do que 1:10 e certificação de instrutores com conhecimento específico sobre TR para crianças e adolescentes.

Neste mesmo período, a Canadian Society for Exercise Physiology $y^{30}$ publicou seu posicionamento a respeito do exercício resistido para crianças e adolescentes. As recomendações são similares as da American Academy of Pediatrics com algumas diferenças: atenção ao desenvolvimento cognitivo, maturidade física e experiência de treinamento; destaque para um ambiente de exercício seguro e livre de perigos; e foco na técnica correta do exercício. Sugerem também variações sistemáticas de treinamento para aperfeiçoar resultados e reduzir o tédio das sessões. Reafirmam a necessidade de supervisão adequada, instrutores qualificados e faz indicações pontuais a respeito das características funcionais que devem ser o produto principal do treinamento para crianças e adolescentes a saber: força muscular, resistência, equilíbrio e coordenação.

No Brasil, um estudo de revisão foi publicado sobre o impacto da atividade física sobre o crescimento de crianças e adolescentes, incluindo o TR. Os autores concluíram que a prática de musculação por crianças pré-púberes, de modo geral, deve ser realizada com cautela e sob supervisão especializada e que esta e outras modalidades esportivas não têm efeitos específicos no sentido de aumentar ou diminuir a altura final ${ }^{31}$.

No ano de 2009 a National Strength and Conditioning Association ${ }^{13}$, publicou atualizações relativas ao treinamento resistido para jovens, destacando benefícios tais como a melhoria nas habilidades motoras e esportivas, o bem-estar psicossocial, a resistência a lesões, a promoção do hábito da prática de exercício ao longo da vida e fatores de proteção relacionados a saúde cardiovascular. Estes indicadores são importantes devido a tendência de redução dos níveis de atividade física com o avanço da idade. Neste contexto, o TR torna-se uma importante alternativa de atividade física estruturada, pois é fato que a capacidade de ganhar força muscular aumenta com a idade e estado de maturação ${ }^{32}$, facilitando a adesão e continuidade das atividades. Os achados corroboram com a hipótese de que o TR bem conduzido pode ser uma forma segura de condicionamento físico, sem interferir de forma negativa nas condições de crescimento e desenvolvimento.

Miller e colaboradores ${ }^{12}$, no ano de 2010 , revisaram as recomendações vigentes na literatura e concluíram que a prescrição do TR para jovens deve contemplar todos os importantes grupos musculares do corpo. Em relação à escolha dos exercícios e intensidade do TR, orientam para a realização de uma a três séries, de seis a quinze repetições, duas a três vezes na semana em dias não consecutivos. A progressão deve ser acompanhada pela execução correta e na medida em que o adolescente se torna mais experiente, exercícios mais complexos podem ser incluídos gradativamente.

Faigenbaum e Myer ${ }^{33}$, em duas revisões publicadas no ano de 2010, observaram que as pesquisas atuais indicam que o TR é uma atividade eficaz e vantajosa para crianças e adolescentes, podendo ser importante no rol de opções oferecidas nesta faixa etária. Os autores reforçam a importância da qualidade e variedade dos exercícios prescritos como sendo fatores primordiais para o sucesso do programa. Destacam que a integração sensata de diferentes métodos de treinamento, junto com a manipulação periódica das variáveis pertinentes à prescrição, são ações imprescindíveis para manter o estímulo, a eficácia, ser desafiante e agradável para os jovens participantes ${ }^{34}$. Convém salientar que estas recomendações, relativas às metodologias de prescrição, são as mesmas da população adulta no intuito de melhorar a aderência e a continuidade nos programas.

Por fim, é possível identificar que os estudos mais recentes apontam para uma tendência em abordar questões associadas à manutenção, diversificação e aderência do TR para jovens. Os manuscritos passam a destacar a importância do TR como complemento das atividades aeróbicas e de condicionamento físico em detrimento às questões que envolvem as lesões e o crescimento, até então tópicos redundantes. Parece não haver mais dúvidas sobre a aplicabilidade, eficácia e segurança do TR para crianças e adolescentes, respeitadas as recomendações vigentes. Vislumbra-se, portanto, uma fase de consolidação do TR para crianças e adolescentes.

\section{CONCLUSÕES: OPORTUNIDADES E EXPECTATIVAS}

Conforme observado, ocorreu uma evolução substancial no número de publicações que abordam a utilização do TR para crianças e adolescentes, a partir do ano de 1990. Apesar de ainda controverso, a literatura atual recomenda a prática do TR para jovens, cujas recomendações mais importantes estão sintetizadas no Quadro 1.

É importante destacar que, tal qual em outros esportes ou modalidades, o problema está na forma como o TR é prescrito 
Quadro 1: Síntese dos principais indicadores relativos ao treinamento resistido para crianças e adolescentes

\begin{tabular}{|ll|l}
\hline $\begin{array}{l}\text { Características dos exercícios } \\
\text { e da sessão }\end{array}$ & $\begin{array}{l}\text { Realizar } 8 \text { a } 12 \text { exercícios estruturais para todo o corpo; } 8 \text { a } 15 \text { repetições; volume moderado; intensidade } \\
\text { moderada a baixa; isotônico; treinamento cardiovascular e de flexibilidade concomitante; } 2 \text { a } 3 \text { vezes na } \\
\text { semana em dias alternados; variar sistematicamente as sessões; utilizar inicialmente o peso do corpo } \\
\text { seguido de equipamentos ou acessórios adequados ergonomicamente. }\end{array}$ \\
\hline Enfatizar & $\begin{array}{l}\text { Amplitude completa; técnica correta; ambiente, materiais e equipamentos adequados e seguros; supervisão } \\
\text { por adulto qualificado; relação instrutor/aluno não maior que 1:10; ingestão adequada de líquidos e alimentos; } \\
\text { priorizar força, resistência, equilíbrio e coordenação. }\end{array}$ \\
\hline Evitar & $\begin{array}{l}\text { Intensidade e volume elevados; caráter competitivo, power lifting e body building; esteróides anabolizantes e } \\
\text { substâncias ilícitas; suplementos de forma arbitrária; equipamentos e ambiente do adulto. }\end{array}$ \\
Mitos & $\begin{array}{l}\text { TR provoca lesões e compromete indicadores antropométricos (peso, estatura), cardiorrespiratórios, } \\
\text { hemodinâmicos e flexibilidade. }\end{array}$ \\
Verdades & $\begin{array}{l}\text { Treinamento rigoroso pode prejudicar a saúde; programas adequados à maturidade física e emocional são } \\
\text { seguros e promovem melhorias nas habilidades motoras, no bem estar psicossocial e na resistência a lesões. }\end{array}$ \\
\hline
\end{tabular}

ou realizado e não propriamente nas suas características enquanto exercício físico. Igualmente, não se pode negar que os padrões aceitáveis impostos pelas convenções sociais relativos aos componentes corporais, aumentam as chances de que crianças e adolescentes adotem estratégias de treinamento além dos parâmetros recomendados. Sobre este aspecto, recomenda-se um constante monitoramento por parte de professores e familiares.

Embora muitos parâmetros analisados indiquem que o TR realizado de forma correta seja seguro, convém destacar que permanecem latentes questões associadas às respostas crônicas do TR em faixas etárias intermitentes aos processos maturacionais, assim como indagações relacionadas à prescrição para as diferentes idades, ergonomia dos equipamentos e ambientes. Estes tópicos se traduzem em oportunidades de pesquisa principalmente para professores de Educação Física, fisioterapeutas e profissionais da medicina do esporte.

Nossa perspectiva é que o tema em questão encontre maior respaldo em estudos futuros que se fazem necessários para elucidar as questões ainda inconclusivas e mais ainda para esclarecer a relação treinamento de força, academias de musculação e adolescentes. $\mathrm{O}$ entendimento destas vertentes pode fazer com que o treinamento de força seja mais uma opção de atividade física segura para crianças e adolescentes.

\section{REFERÊNCIAS}

1. Da Silva CC, Goldberg TBL, Teixeira AS, Marques I. O exercício físico potencializa ou compromete o crescimento longitudinal de crianças e adolescentes? Mito ou verdade? Rev Bras Med Esporte. 2004;10(6):520-524.

2. Guy JA, Micheli LJ. Strength training for children and adolescents. J Am Acad Orthop Surg. 2001;9(1):29-36.

3. Falk B, Tenenbaum $\mathrm{G}$. The effectiveness of resistance training in children. A meta-analysis. Sports Med. 1996;22(3):176-86.

4. Berhardt DT, Gomez J, Johnson MD, et al. Committee on Sports Medicine and Fitness. Strength training by children and adolescents. Pediatrics. 2001;107(6):1470-2

5. Malina RM. Weight training in youth-growth, maturation, and safety: an evidence-based review. Clin J Sport Med. 2006;16(6):478-87.

6. Stricker PR. Sports training issues for the pediatric athlete. Pediatr Clin North Am. 2002;49(4):793-802.

7. Falk B, Eliakim A. Resistance training, skeletal muscle and growth. Pediatr Endocrinol Rev. 2003;1(2):120-7.

8. Da Silva CC, Teixeira AS, Goldberg TBL. Sport and its implications on the bone health of adolescent athletes. Rev Bras Med Esporte. 2003;9(6):426-432

9. Karsenty $\mathrm{G}$. Bone endocrine regulation of energy metabolism and male reproduction. C R Biol. 2011;334(10):720-4.
10. Karsenty G. The mutual dependence between bone and gonads. J Endocrinol. 2012;213(2):107-14.

11. Shi YC, Baldock PA. Central and peripheral mechanisms of the NPY system in the regulation of bone and adipose tissue. Bone. 2012;50(2):430-6.

12. Miller MG, Cheatham CC, Patel ND. Resistance training for adolescents. Pediatr Clin North Am. 2010;57(3):671-82.

13. Faigenbaum AD, Kraemer WJ, Blimkie CJ, Jeffreys I, Micheli LJ, Nitka M, Rowland TW. Youth resistance training: updated position statement paper from the national strength and conditioning association. J Strength Cond Res. 2009;23(5 Suppl):S60-79.

14. American College of Sports Medicine. Progression models in resistance training for healthy adults. Med Sci Sports Exerc. 2009;41(3):687-708.

15. McCambridge TM, Stricker PR. Strength training by children and adolescents. American Academy of Pediatrics Council on Sports Medicine and Fitness. Pediatrics 2008;121(4):835-40.

16. Ratel S. High-intensity and resistance training and elite young athletes. Med Sport Sci. 2011;56:84-96.

17. Webb DR. Strength training in children and adolescents. Pediatr Clin North Am. 1990;37(5):1187-210.

18. Risser WL. Musculoskeletal injuries caused by weight training. Guidelines for prevention.Clin Pediatr (Phila). 1990;29(6):305-10. 
19. Risser WL. Weight-training injuries in children and adolescents. Am Fam Physician. 1991;449(6):2104-8.

20. Blimkie CJ. Resistance training during pre- and early puberty: efficacy, trainability, mechanisms, and persistence. Can J Sport Sci. 1992;17(4):264-79.

21. Blimkie CJ. Resistance training during preadolescence. Issues and controversies. Sports Med. 1993;15(6):389-407.

22. Payne VG, Morrow JR Jr, Johnson L, Dalton SN. Resistance training in children and youth: a meta-analysis. Res Q Exerc Sport. 1997;68(5):80-8

23. Alleyne JM. Safe exercise prescription for children and adolescents. Pediatr Child Health. 1998;3(1):337-42.

24. Faigenbaum AD. Strength training for children and adolescents Clin Sports Med. 2000;19(3):593-619.

25. Washington RL, Bernhardt DT, Gomez J, et al. Organized sports for children and preadolescents. Pediatrics. 2001;107(6):1459-62.

26. Benjamin HJ, Glow KM. Strength training for children and adolescents: what can physicians recommend? Phys Sportsmed. 2003;31(9):19-26

27. Myer GD, Wall EJ. Resistance training in the young athlete. Operative techniques in sports Medicine. 2006;14(3):218-230.
28. Benson AC, Torode ME, Fiatarone Singh MA. Effects of resistance training on metabolic fitness in children and adolescents: a systematic review Obes Rev. 2008;9(1):43-66.

29. Vaughn JM, Micheli L. Strength Training Recommendations for the Young Athlete. Physical medicine and rehabilitation clinics of North America. 2008;19(3):235-241.

30. Behm DG, Faigenbaum AD, Falk B, Klentrou P. Canadian Society for Exercise Physiology position paper: resistance training in children and adolescents. Appl Physiol Nutr Metab. 2008;33(3):547-61.

31. Alves C, Lima R. Impacto da atividade física e esportes sobre o crescimento e puberdade de crianças e adolescentes. Rev Paul Pediatr. 2008;26(4):383-91

32. Behringer M, Vom Heede A, Yue Z, Mester J. Effects of resistance training in children and adolescents: a meta-analysis. Pediatrics. 2010;126(5):1199-210.

33. Faigenbaum AD, Myer GD. Pediatric resistance training: benefits, concerns, and program design considerations. Curr Sports Med Rep. 2010;9(3):161-8.

34. Faigenbaum AD, Myer GD. Resistance training among young athletes: safety, efficacy and injury prevention effects. Br J Sports Med. 2010;44(1):56-63. 